\title{
Homicidio Policial, Víctimas e Impunidad. Tres nociones para pensar la Violencia Estatal en su radicalidad.
}

\author{
Homicides Committed by the Police, Victims and Impunity. Three notions to tackle \\ State Violence in its Radicalism.
}

\section{Chena, Marina; Fernández, Natalia; Miretti, Jeremías; Molas y Molas, María; Petiti, Yanina; Valenzuela, Héctor ${ }^{1}$}

Resumen. Son diversos los puntos críticos que nos interpelan en el quehacer profesional como psicólogos/as frente a las políticas de seguridad de un Estado Policial fuertemente represivo y que tiene a los jóvenes de sectores populares como blanco predilecto en la Provincia de Córdoba. Nos propusimos construir un dispositivo específico de intervención que dio origen al Equipo de Acompañamiento a Familiares de Jóvenes asesinados por la Policía. En este camino de consolidación del dispositivo, resultan relevantes los anclajes conceptuales para pensar la intervención. En este trabajo nos proponemos problematizar tres nociones que insisten ser pensadas: homicidio policial, el concepto de víctima y la cuestión de la impunidad que prevalecen incluso en un estado democrático. Estas categorías operan como ejes que orientan la presente reflexión y buscan dar cuenta del proceso de construcción de nuestros fundamentos tanto ético-políticos como teórico-prácticos.

\footnotetext{
${ }^{1}$ Chena, Marina. Lic. en Psicología- Universidad Nacional de Córdoba. Colegio de Psicólogos de la Provincia de Córdoba-Argentina. marinachena@gmail.com.

Fernández, Natalia. Lic. en Psicología- Universidad Nacional de Córdoba. Secretaría de Derechos Humanos y Pluralismo Cultural de la Nación-Delegación Córdoba-Argentina. naty.fernandez1986@gmail.com.

Miretti, Jeremías. Lic. en Psicología- Universidad Nacional de Córdoba. Colegio de Psicólogos de la Provincia de Córdoba-Argentina. jeremiasmiretti@gmail.com.

Molas y Molas, María. Lic. en Psicología- Universidad Nacional de Córdoba. Secretaría de Derechos Humanos y Pluralismo Cultural de la Nación-Delegación Córdoba-Argentina. Mariamolas74@ gmail.com.

Petiti, Yanina. Lic. en Psicología- Universidad Nacional de Córdoba. Observatorio de Derechos Humanos de la Secretaría de Extensión y Vinculación de la Universidad Nacional de Córdoba- Argentina. yaninapetiti@gmail.com.

Valenzuela, Héctor. Lic. en Psicología- Universidad Nacional de Córdoba. Secretaría de Derechos Humanos y Pluralismo Cultural de la Nación-Delegación Córdoba-Argentina. valenzuelacomerc@ hotmail.com.
}

Debates actuales en Psicología y Sociedad. ISSN en línea: 2591-524X

Año II, N² 2, 2019. Pág. 17-29.

DOI: 10.22529/daps.2019.2(2)02 / Recibido: 13-08-2018 / Aprobado: 2-1-2019.

Artículo publicado bajo Licencia Creative Commons Atribución-NoComercial-SinDerivar.

(C) Universidad Católica de Córdoba. 
Palabras clave: Violencia estatal, homicidio policial, víctimas, impunidad.

\begin{abstract}
There are several critical points which affect our professional work as psychologists when facing the severely repressive security measures from a Police State which mainly targets young people from the lowest income sectors of society of Córdoba Province. We set the goal of coming up with a specific intervention measure. That goal resulted in the creation of the Support Team for Family Members of Youngsters Murdered by the Police. When pursuing the consolidation of that measure, conceptual anchors become relevant to both think and understand the intervention. Our goal in this project is to problematize three notions that must be tackled: homicides committed by the police, the victim as a concept, and the impunity that prevails even during a democratic state. These categories operate as axes orientating the current reflection and intend to render account of the construction process of our ethical-political fundamentals as well as the theoretical and practical ones.
\end{abstract}

Keywords: State violence, homicides committed by the police, victim, impunity. 


\section{Posicionamientos y consideraciones}

El trabajo de acompañamiento en casos de homicidio policial es una experiencia novedosa que cuenta con escasos antecedentes locales. Como tantas otras problemáticas, existen diversos posicionamientos, tanto teóricos-prácticos como éticos-políticos, que orientan el abordaje en tan compleja labor. Resulta por lo tanto de fundamental importancia explicitar cuáles son algunos de los puntos críticos que nos interpelan en el quehacer, reflejan nuestro posicionamiento como Equipo y hacen a la delimitación conceptual del campo de problemáticas al que se destina la intervención. Estos son: el concepto de homicidio policial, la noción de víctima y la cuestión de la impunidad.

\section{a. Homicidio policial}

Nos parece importante realizar un breve recorrido que se inicia con el concepto de gatillo fácil, el cual permitió un posicionamiento político-militante de gran envergadura y posibilitó a su vez visibilizar en la esfera pública casos de jóvenes asesinados por las fuerzas de seguridad. En los últimos tiempos nos aproximamos a definiciones teóricas que se anclan en conceptos tales como uso letal de la fuerza o violencia estatal ejercida por las fuerzas de seguridad a los fines de delimitar el universo a los casos más extremos y radicales. Creemos relevante destacar que cuestionamos fuertemente el concepto de violencia institucional que también comenzó a difundirse ampliamente en los últimos años en todo el territorio nacional ya que, siguiendo los aportes de la socióloga Daroqui (2016) consideramos dicho concepto desdibuja el sujeto y oculta la participación del Estado, que es justamente el perpetrador de estas violencias. El Estado y sus funcionarios son los actores de la violencia estatal y por ello se constituye en violaciones a los derechos humanos. "En los territorios urbanos donde la policía se despliega no podemos hacer desaparecer al Estado, es la responsabilidad del estado en su dimensión gubernamental” (p. 17).

El Grupo de Estudio sobre el Sistema Penal y Derechos Humanos del Instituto de Investigaciones Gino Germani de la Facultad de Ciencias Sociales de la Universidad de Buenos Aires, expresa que

(...) la violencia institucional hace referencia a un concepto amplio y a la vez difuso, en cierto modo inespecífico que requiere fuertes desagregaciones. En principio que se separe lo público de lo privado y dentro de lo público que escinda aquello que se inscribe en el marco de las violencias de las agencias de seguridad y custodia, de las producidas por otras instituciones. Sin esta desagregación, el concepto de violencia 
institucional no permite distinguir la articulación entre la dimensión estatal y la de los derechos humanos en clave de violencia. (s/f en Daroqui, 2016, p. 19)

En este recorrido conceptual nos parece interesante aproximarnos al concepto de violencia estatal ejercida por las fuerzas de seguridad para finalmente referirnos de manera específica a los casos de homicidio policial. Continuando con los aportes de la autora citada, al destacar que se trata de un homicidio nos sitúa en el marco de un delito, un crimen, y como tal tiene un autor, en este caso la policía; nos circunscribe a esa fuerza de seguridad debido a que es la que se expande en los territorios, en especial en los sectores populares. Por otro lado, al ser un homicidio el Estado tiene la responsabilidad de investigar y juzgar a los responsables, con el agravante de ser funcionarios públicos y de la utilización de armas de fuego. En este sentido los hechos juzgados no tendrían los habituales atenuantes que suelen utilizar tanto la fuerza de seguridad como los medios de comunicación y el poder judicial en estos casos, donde son presentados como "enfrentamientos en el marco de la persecución del delito" y en los cuales sorpresivamente los únicos muertos son los supuestos delincuentes. De esta manera al nombrarlos como homicidios policiales dejamos de colaborar con los eufemismos que el poder represor impone.

Homicidio Policial, sabemos los responsables, los autores de estos crímenes, los victimarios. Entonces cabe preguntarse ¿a quién mata el Estado en democracia?, ¿quiénes son las víctimas de estos crímenes? Homicidios policiales perpetrados de manera sistemática y generalizada hacia determinados grupos sociales, jóvenes varones de sectores populares.

\section{b. Víctimas}

La categoría de víctima, propia del campo jurídico, remite al hecho de que alguien ha sufrido un daño. Según la definición surgida de la Declaración sobre los Principios Fundamentales de la Justicia para víctimas del delito y del abuso del poder, de la Asamblea General de la Organización de las Naciones Unidas se establece que

Víctimas de delitos. Se entenderá por "víctimas" las personas que, individual o colectivamente, hayan sufrido daños, inclusive lesiones físicas o mentales, sufrimiento emocional, pérdida financiera, o menoscabo sustancial de los derechos fundamentales, como consecuencia de acciones u omisiones que violen la legislación penal vigente en los Estados miembros, incluida la que proscribe el abuso de poder. Podrá considerarse 
"víctima" a una persona con arreglo a la presente Declaración, independientemente de que se identifique, aprehenda, enjuicie o condene al perpetrador e independientemente de la relación familiar entre el perpetrador y la víctima. En la expresión "víctima" se incluye además en su caso, a los familiares o personas a cargo que tengan relación inmediata con la víctima directa y a las personas que hayan sufrido daños al intervenir para asistir a la víctima en peligro, o para prevenir la victimización. Las disposiciones de la presente Declaración serán aplicables a todas las personas sin distinción alguna ya sea de raza, color, sexo, edad, idioma, religión, nacionalidad, opinión política o de otra índole, creencias o prácticas culturales, situación económica, nacimiento o situación familiar, origen étnico o social o impedimento físico. (Organización de las Naciones Unidas, 1985)

Este concepto, ha tenido una importancia diferencial a lo largo del tiempo. Es en las últimas décadas que las víctimas han ocupado un lugar central, tanto en el plano teórico, como en las políticas públicas, en el activismo, en los medios de comunicación y en la opinión pública.

Distintos autores (Garland, 2005; Pita, 2010; Bermúdez, 2008, 2016; Rodríguez Alzueta, 2014) coinciden en mostrar de qué modo la definición respecto de lo que es una víctima, lejos de resolverse mediante una tipificación penal o jurídica, responde a complejos procesos sociales de atribución de sentido -dimensión simbólica-, disputas por esos sentidos dimensión política, exigencias de justicia -dimensión ética-, la institución de una cierta figura o imagen de víctima-dimensión estética, etc.

Ciertamente el sentido hegemónico y a partir del cual se ha configurado la idea de víctima en el campo jurídico es el de inocencia. Tal como mencionamos más arriba, víctima desde esta perspectiva- es quien ha sufrido un daño, pero, siguiendo a Pitch (2009) "para ser víctimas merecedoras no basta con haber sufrido algo de parte de alguien, sino haber hecho todo lo posible para no serlo y poder demostrar no haber estado en condición de poder hacer algo para evitarlo" (p.67).

Estas víctimas merecedoras e inocentes, son el producto de una construcción social y política que las sitúan en el centro de la escena. Son convocadas a dar testimonios, se les ofrece protagonismo mediático, en ellas se fundan las demandas de "mano dura", incremento de las penas u otras medidas punitivas y se universaliza un sentimiento de empatía hacia su sufrimiento. Esta operatoria es posible en razón de una generalización atribuida a la 
victimización. Tal como describen Pitch (2009), Garland (2005) entre otros autores, en los últimos años la condición de víctima es posible de hacerse extensiva a toda la sociedad, bajo la premisa de que a cualquiera puede pasarle. Así, queda configurada un estatuto de víctimas potenciales según el cual es posible homogeneizar -al menos idealmente- un conjunto social, las víctimas actuales y potenciales; una causa, el delito común abordado desde la matriz de la inseguridad y una estrategia punitiva de control y prevención de esos delitos.

No obstante, como señalamos anteriormente, este sentido homogéneo y unívoco de víctima es ilusorio, en la medida que otros actores disputan -aún desde lugares periféricos de la enunciación social- otros sentidos y politizan la concepción aséptica de las víctimas inocentes de la inseguridad urbana. Las víctimas de la violencia estatal, usualmente son víctimas "no inocentes" (Bermúdez, 2008). En estos casos, el concepto hegemónico-jurídico no resulta fértil para comprender los procesos sociales de construcción de las víctimas. En primer lugar, no son universalizables, no somos todos como sociedad los destinatarios del daño, sino que está diferencialmente dirigido hacia ciertos sectores sociales, a un grupo etario y privilegiadamente a un género: jóvenes varones de sectores populares. En segundo lugar, el daño que los instituye en víctimas lo producen agentes del estado, miembros de las fuerzas de seguridad. Por esta razón, la posibilidad de articular un discurso "desde arriba" es decir desde el estado, acerca de la necesidad de una condena punitiva sobre los perpetradores, se ve obstaculizada o directamente negada. Son los familiares quienes politizan la condición de víctima, quienes las posicionan en el escenario público y quienes expresan y vehiculizan la demanda de justicia legítima también para ellas. Plantea Calzado (2014) que "si las instituciones policiales construyeron esas muertes como legítimas, los padres, los hermanos deben restituir la memoria de la "buena vida" de los muertos" (p.43). Es necesario humanizarlos nuevamente, volver humana esa vida a "quien cualquiera puede dar muerte" según palabras de Agamben (1998-2006). La figura de los familiares se torna central en el análisis de los modos en que sus demandas de justicia contribuyen a disputar sentidos hegemónicos a la idea de las víctimas inocentes como categoría omnicomprensiva.

En este sentido cabe preguntar, ¿Cuáles son los sentidos que toma, en los casos de jóvenes asesinados por la policía y el pedido de justicia por parte de sus familiares, la cuestión de la impunidad? 


\section{c. La impunidad, una problemática situada}

En primer lugar, es importante señalar que el concepto de impunidad no se restringe a un análisis propiamente técnico o jurídico, ni su discusión o resolución se define sólo desde la perspectiva del derecho. Las consecuencias del fenómeno de la impunidad alcanzan y afectan al campo de lo social en su conjunto.

"La Impunidad significa ausencia de justicia y de verdad, y la democracia pasa a jugar un papel insignificante o inexistente. Tiene efectos que configuran un conjunto de secuelas en los implicados y en la sociedad que lesionan, causan daños y fracturas en el lazo social, perturbando y desequilibrando la estabilidad individual y colectiva". (Macedo, 2008, p.76)

Así mismo, para la elucidación de nuestro caso en particular, los jóvenes cordobeses asesinados por la policía, es necesario y aun ineludible situar esta problemática en nuestras circunstancias políticas, históricas y territoriales. Tal como expresa Bottinelli (2005) "la impunidad se ha visto asociada, más que con teorías jurídicas, con situaciones históricas que marcaron profundamente a las comunidades latinoamericanas. La impunidad en América Latina más que un concepto, es una realidad histórica que ha configurado el presente de sus sociedades" (p.186).

Para sintetizar, diremos junto con Ventura Robles (2005) que "el concepto "impunidad" no describe, en sentido estricto, un asunto legal, sino un fenómeno de dimensiones legales, sociales, culturales, psicológicas y hasta económicas”(p.12). Así, se configura no sólo como la ausencia o inexistencia de castigo, de responsabilidad penal ante una infracción de la ley, sino como un acto de violencia en sí misma generada por la inaccesibilidad del derecho a la justicia. En este sentido, opera también como un dispositivo de control social que internaliza aquella idea de que no habrá cumplimiento del castigo para los culpables instalando así una naturalización de las relaciones de poder y de sometimiento. La impunidad entonces se instituye como una cultura, constituyéndose en un conjunto de instituciones, hábitos, creencias, actitudes y comportamientos que eternizan la violencia, las injusticias, los delitos y las violaciones a los derechos humanos.

c. 1. Continuidades del accionar represivo e impunidad: jóvenes cordobeses asesinados por las fuerzas de seguridad. 
Barrault (2016), sitúa dentro de un conjunto de reflexiones algunos puntos para elucidar sobre este tema. En primer lugar, la reactualización que adquiere dicha problemática asociada inicialmente al Terrorismo de Estado en Argentina, en los casos de jóvenes asesinados por las fuerzas de seguridad. En este sentido Segato (2016) plantea que

La construcción del otro antagónico en nuestra historia nacional es letal. La generaron aquellos que solamente estaban interesados en una masacre. En un genocidio. Esa construcción de antagonismo, que además es muy característica de la Argentina, el otro como un antagonista para el que no hay lugar dentro de una sociedad común. (párr. 8)

La construcción de esa otredad en nuestros casos está planteada por una infinidad de características que los discursos sociales se encargan de estigmatizar. Los asesinatos que perpetra la policía de Córdoba son en la generalidad de los casos, sobre jóvenes, varones, de procedencias populares, territorialmente localizables en zonas en las que lo constante es la ausencia del Estado en su carácter de garante y su omnipresencia en su fase represiva.

De allí que los medios de comunicación se encarguen de reportar aquellos episodios siempre velados por la potencial culpabilidad de la víctima. "Enfrentamientos", "tiroteo", "fuga", "comisión de delito" son algunas de las adjetivaciones con las que se acompaña la crónica sobre los asesinatos de estos jóvenes. Inclusive las mismas fuerzas de seguridad gestionan de formas múltiples la implantación de evidencias en los "procedimientos", que pretenden en un doble movimiento, culpabilizar a la víctima y desresponsabilizar a los asesinos.

Así, siguiendo con la línea que plantea la continuidad entre el Terrorismo de Estado y los casos de homicidio policial en democracia en nuestra provincia, se reconstruye nuevamente el "algo habrán hecho". Se naturaliza con fuerza la idea de que esa muerte está justificada no ya sólo porque sería en respuesta a una "infracción", sino, además, porque es ejecutada sobre una población que está en los márgenes de la sociedad, sobre los y las que recae un discurso de inutilidad social. En este sentido afirma Segato (2016) que estos son “muertos merecedores de morir". Haremos una breve puntualización aquí: los medios de comunicación son uno de los principales transmisores de lo que se denomina la "Pedagogía de la Crueldad", esa espectacularización del sufrimiento que tiene como principal objetivo "enseñar que ese horizonte de sufrimiento es la normalidad" (párr.11). 
Sobre esta caracterización, insiste la idea de una impunidad justificada para los casos de estos jóvenes, que despliega su fuerza en el orden de lo moral construyendo criterios de valoración y percepción social de la población con respecto a las víctimas a partir de la estigmatización, consiguiendo el aislamiento y la desacreditación de estas personas. Hay en ese mensaje, emanado de los círculos de poder y dominación, un proyecto social, un destino ideológico para estas poblaciones que sentencia un devenir marginal, una vida precaria. Se podría pensar aquí en la noción de "vida desnuda" propuesta por Agamben (1998) que, como retoma Giorgi (2008) “refiere siempre a una 'zona de indistinción' entre la vida humana socialmente reconocible y la vida 'meramente biológica', es decir la vida despojada de las marcas que la vuelven objeto de protección y reconocimiento por parte de un orden jurídico y político dado (...) donde la legibilidad social de un cuerpo se interrumpe” (p. 48). Vidas, desprovistas de reconocimiento social, económico, político que reflejan la degradación de lo social propia de la era neoliberal.

En el transcurso de nuestro trabajo, esto es, como psicólogas y psicólogos que acompañamos a familiares de jóvenes asesinados por la policía de Córdoba, hemos presenciado los innumerables efectos con los que opera la impunidad, en un movimiento que es dialéctico entre las afectaciones colectivas, comunitarias y sociales y el propio malestar psicológico, subjetivo y singular.

Diversos autores/as (Aguiar, 1993; Mejía Correa, 2005; Bottinelli, 2005; Gómez Isa, 2011) se han encargado de pormenorizar los sentimientos que emergen de las situaciones de impunidad, tales como frustración, angustia, miedo, culpa, rabia, aislamiento, impotencia, desconfianza y desamparo entre otros. Como dirá Barrault (2016) estos son "una serie de sentimientos ante el avasallamiento en una relación de poder. No es solo además una relación de poder sino la percepción de lo injusto de esta relación. Implica cierta relación con lo público por su expresa presencia o su insistente ocultamiento" (p.6).

Entendemos que los efectos de la impunidad pendulan entre las tensiones de lo singular y lo colectivo. La experiencia de nuestro equipo nos lleva entonces a posicionarnos desde un enfoque psicosocial que tenga a lo social comunitario como el espacio privilegiado de tramitación de estas afectaciones. La construcción de redes de sostén que permitan el pasaje a lo público del dolor producido por la pérdida de un familiar, amigo, vecino, la reconstrucción del lazo social, asentado en una cultura de derechos humanos y su ejercicio ciudadano, la transformación de las instituciones de justicia y la garantía del derecho por parte 
de los familiares a la verdad, la justicia y la reparación (Gómez Isa, 2011) son pilares fundamentales para la lucha contra la impunidad.

\section{Referencias Bibliográficas}

Agamben, G. (1998-2006). Homo sacer. El poder soberano y la nuda vida. I. Valencia, España: Pre textos.

Aguiar, E. (1993). Efectos psicosociales de la impunidad. Recuperado de http://www.apdhargentina.org.ar/sites/default/files/u6/Efectos\%20psicosociales\%20de\%201a\%20impu nidad.pdf

Barrault, O. (23 de septiembre de 2016). 25 puntos sobre la Impunidad. Trabajo presentado en el Encuentro de Presentación de la experiencia del Equipo de acompañamiento psicológico a testigos víctimas, familiares y querellantes en la Megacausa por crímenes de Lesa humanidad, La Perla -la Rivera. Municipalidad de Córdoba. Secretaría de Salud. Dirección de Atención Primaria de la Salud. Inédito. Recuperado de https://www.academia.edu/31594061/25_puntos_sobre_la_impunidad.pdf

Bermúdez, N. (2008). "Merecer la muerte" Una antropología sobre las moralidades y las prácticas políticas en torno a las muertes violentas (Córdoba-Argentina). Trabajo presentado en IX Congreso Argentino de Antropología Social. Facultad de Humanidades y Ciencias Sociales. Universidad Nacional de Misiones, Posadas. Recuperado de https://www.aacademica.org/000-080/63.

Bermúdez, N. (2016). De morir como perro a me pinto sólo cuatro uñas. Una mirada antropológica sobre crueldad, moralidad y política en muertes vinculadas a la violencia institucional en Córdoba (Argentina). Publicar en Antropología y Ciencias Sociales, $14(20)$ Recuperado de http://ppct.caicyt.gov.ar/index.php/publicar/article/view/7181/8494.

Bottinelli, M.C. (2005). La impunidad como crimen de lesa humanidad. San José, Costa Rica: Instituto Interamericano de Derechos Humanos. Recuperado de https://biblioteca.iidhjurisprudencia.ac.cr/index.php/documentos-en-espanol/prevencion-de-la-tortura/1227la-impunidad-como-crimen-de-lesa-humanidad/file

Calzado, M. (2014). Violencia, víctimas y mediatización. Un acercamiento empírico a la 
conformación de subjetividades en las sociedades de seguridad. Delito y Sociedad, 37(1), 41-59. Recuperado de https://bibliotecavirtual.unl.edu.ar/ojs/index.php/DelitoYSociedad/article/viewFile/554 $\underline{1 / 8275 .}$.

Daroqui, A. (1 de diciembre de 2016) La cárcel de la democracia: una interpelación a la cuestión de los Derechos Humanos del presente. Charla organizada por el Observatorio de Derechos Humanos de la Secretaría de Extensión Universitaria de la Universidad Nacional de Córdoba. Recuperado de https://www.unc.edu.ar/sites/default/files/Publicaci\%C3\%B3n\%20charla\%20Alcirafinal.pdf

Garland, D. (2005). La cultura del control. Crimen y orden social en la sociedad contemporánea. Barcelona, España: Gedisa.

Giorgi, G. (2008). Lugares comunes: “vida desnuda” y ficción. Grumo, 7, 48-55. Recuperado de http://www.redkatatay.org/sitio/talleres/grumo.pdf.

Gómez Isa, F. (noviembre de 2011). El fenómeno de la impunidad: luces y sombras en América Latina. Revista de Derechos Humanos Dfensor. 11, 42-48. Recuperado de http://cdhdf.org.mx/wp-content/uploads/2014/05/dfensor_11_2011.pdf.

Macedo, M. (2008). Reflexiones sobre la impunidad. Revista Regional de Trabajo Social. 22(43), 76-77. Recuperado de http://www.revistatrabajosocial.com.

Mejía Correa, M.P. (2005). Efectos psíquicos de la impunidad. El retorno del espectáculo. Desde El jardín de Freud, 5, 268-272. Recuperado de http://revistas.unal.edu.co/index.php/jardin/article/view/8417/9061.

Organización de las Naciones Unidas (29 de noviembre de 1985). Principios fundamentales de justicia para las víctimas del delito y del abuso de poder. Resolución 40/34 de la $\begin{array}{llll}\text { Asamblea } & \text { General, } & \text { anexo. }\end{array}$ http://www.ohchr.org/SP/ProfessionalInterest/Pages/VictimsOfCrimeAndAbuseOfPo wer.aspx.

Pita, (2010). Formas de morir y formas de vivir el activismo contra la violencia policial. Buenos Aires, Argentina: Del Puerto/CELS.

Pitch, T. (2009). La sociedad de la prevención. Buenos Aires, Argentina: Ad hoc. 
Rodríguez Alzueta, E. (2014). Temor y control. La gestión de la inseguridad como forma de gobierno. Buenos Aires, Argentina: Futuro Anterior Ediciones.

Segato, R. (18 de septiembre de 2016). La construcción del otro como antagónico es letal. Enredando, Recuperado de http://www.enredando.org.ar/2016/09/18/rita-segato-laconstruccion-del-otro-como-antagonico-es-letal/.

Ventura Robles, M. (2005). La jurisprudencia de la Corte Interamericana de derechos humanos en materia de acceso a la justicia e impunidad. Trabajo presentado en el Taller Regional sobre Democracia, Derechos Humanos y Estado de Derecho. Oficina del Alto Comisionado de las Naciones Unidas para los Derechos Humanos. Costa Rica.

Recuperado

de http://www2.ohchr.org/spanish/issues/democracy/costarica/docs/PonenciaMVentura.d oc. 